\title{
El lenguaje jurídico y la divulgación del Derecho*
}

Cristina Cázares Sánchez**

RESUMEN La complejidad de divulgar al derecho para dar respuesta a los cuestionamientos sociales podría ser la variable de lenguaje técnico, que los expertos del derecho dominan y traducen desde la hermenéutica como punto de partida para difundirlo, existiendo una disociación entre lo comunicado por los expertos jurídicos y entendido por el público en general. Una de las propuestas para acercar a las Facultades de Derecho y al público en general es incluir a la divulgación del derecho como una competencia dentro de los programas de estudios.

Palabras clave: Divulgación del Derecho, Enseñanza del Derecho.
ABSTRACT: A reflection on the complexity to achieve an adequate dissemination of law is presented, starting from the indication of hermeneutics as an indispensable variable for the beginning of the dissemination from the Faculties of Law to society in general with the aim of creating spaces of connection with social needs, responding to various questions raised by the social sectors and thereby promoting a culture of legality.

Keywords: Divulgation of Law, Teaching of Law.

SUMARIO: Introducción. 1. La hermenéutica y el texto jurídico. 2. Hermenéutica y divulgación del derecho. 3. Información y divulgación en los medios de comunicación: ¿la divulgación del Derecho es exclusiva de los expertos jurídicos o los periodistas pueden participar en ella? 4. La humildad académica. Conclusión. Fuentes de Consulta.

\section{Introducción}

El Derecho como ciencia posee un código de lenguaje funcional que permite la reproducción de su propio sistema; los actores involucrados, también llamados operadores jurídicos pueden entender, como una suerte de iluminados o grupo de élite, los considerandos de una sentencia, se les presenta muy clara la diferencia entre la verdad jurídica frente a la verdad social difundida por los medios de comunicación, que el común de las personas percibe en un lenguaje complejo y que implica en algunas ocasiones como se han vistas como injusticias sociales.

\footnotetext{
* Artículo recibido el $1^{\circ}$ de mayo de 2020 y aceptado para su publicación el 21 de mayo de 2020.

** Profesora de Carrera en la Facultad de Derecho de la Universidad Nacional Autónoma de México.
} 
Para los expertos jurídicos, resultan obvios, los efectos de una cláusula rebus sic stantibus que permite no cumplir con las obligaciones contractuales cuando existan situaciones imprevistas, y así un largo etcétera de ejemplos, entre otros tantos conceptos que estructuran el Derecho, sin embargo, ese lenguaje técnico e indescifrable transforma realidades sociales, rompe la barrera de la Ciencia Jurídica para introducirse en la vida real, es decir en la vida social e individual de las personas cuyo código de lenguaje dista mucho del utilizado por los operadores jurídicos.

Este trabajo surge como una reflexión después de cursar el Primer Diplomado de Divulgación de las Humanidades y las Ciencias Sociales (2019) organizado por la Coordinación de Humanidades de la Universidad Nacional Autónoma de México -UNAM-, el objetivo es evidenciar al lenguaje técnico como el principal obstáculo para la divulgación del derecho en los actores sociales que precisamente se convierten en los sujetos de Derecho. Este ensayo que retoma el corpus teórico-conceptual y metodológico de la hermenéutica que permite a los operadores jurídicos conocer el Derecho, pero impide darlo a conocer a un público más heterogéneo, es decir a la sociedad en general, en el entendido que las normas jurídicas van dirigidas de forma abstracta a diversos sectores de la sociedad, que no necesariamente tienen un grado de escolaridad.

Entre los objetivos del presente trabajo se encuentra reflexionar si la divulgación del Derecho que es por excelencia texto implica necesariamente leer para conocerlo, si solo se puede divulgar a través de la palabra escrita y si es tarea únicamente de los expertos o bien, puede terceros ajenos a los operadores jurídicos, dedicarse a ello, es decir, si los periodistas, pueden hacer divulgación del derecho, de la misma manera que existe el periodismo científico.

La primera parte se centra en una reflexión sobre la hermenéutica y el lenguaje que utilizan los sujetos expertos para dar a conocer el derecho a los otros, para ello se utilizan autores clásicos que evidencian la subjetividad en la interpretación de los hechos y su comunicación. En la segunda parte, los retos de la divulgación del lenguaje jurídico para un público general y, por último, una breve propuesta para conjuntar la hermenéutica propia del Derecho y la divulgación del Derecho hacia el fomento de una cultura de la legalidad.

\section{La hermenéutica y el texto jurídico}

Cabe señalar que el tema de hermenéutica, si bien es un tema obligatorio en la maestría y doctorado en Derecho, son orientados en términos de interpretación del Derecho, hasta ahora no se presentan desde la perspectiva de la divulgación, del reto interpretativo que implica, como se mencionó en el Primer Diplomado de Divulgación de las Humanidades y las Ciencias Sociales, en el módulo 1, Dr. Enrique Diaz Álvarez en la sesión 7 (23/04/2019).

De tal manera, que me hizo reflexionar acerca de lo que el Derecho construye como realidades jurídicas completamente lógicas y llenas de sentido jurídico pero que implican un sinsentido para la sociedad, que las percibe 'sin explicación lógica' pensando de inmediato que se trata de actos de corrupción porque en el imaginario colectivo, los jueces debiesen actuar de la manera en que la sociedad espera que actúen y en el sentido jurídico, los jueces actúan como el Derecho espera que actúen. 
Lo anterior, me ha llevado a cuestionarme si actualmente en la Ciencia Jurídica existe una disrupción entre Derecho y la Divulgación, entre lo que interpretamos los operadores jurídicos y lo que interpreta la sociedad a la que se dirige la norma, en algún momento ¿se cambia el sentido?

El divulgador es un intérprete y al estilo de Hermes juguetón, dota de sentido a los mensajes para comunicarlos a otros y estos se comprendan ¿podrá haber un malentendido en esta comunicación?

La Hermenéutica es una actividad de interpretación de textos tomados en su contexto. "Interpretar una obra es descubrir el mundo al que ella se refiere en virtud de su disposición, de su género y estilo" (Ricoeur, 1984, cit por Arráez et al., 2006: 174).

Schleiermarcher concibe el lenguaje como:

(a) un acuerdo originario entre el hombre y el mundo, (b) una actividad racional y voluntaria, (c) una producción espontánea y sensible del sujeto que prevalece en la práctica de los hechos históricos en cuanto contiene en su estructura interna de manera innata, la visión del mundo que ha generado, permitiendo al individuo protagonizar la historia de la humanidad. (Ricoeur,1984, cit por Arráez et al., 2006: 174)

Entonces, comunicar el sentido jurídico es una actividad de interpretación de textos que son leídos por operadores que conocen los códigos del lenguaje jurídico y les dotan de su propio estilo, de la visión del mundo que les genera por el solo hecho de vivir, de poseer la calidad de seres humanos dentro de un entorno determinado.

Según Habermas la racionalidad comunicativa: "la estrecha relación que existe entre saber y racionalidad permite sospechar que la racionalidad de una emisión o de un manifestación depende de la fiabilidad del saber que encarnan" (Habermas, 1986: 24), lo cual permitirá llevar a una serie de interacciones entre los saberes y la racionalidad del sistema para llegar a un consenso acerca de la dotación de sentido, pero el consenso de sentido solo puede darse entre iguales, es decir, entre quienes entiendan el mismo código y no es el caso de la sociedad en general.

Para Luhmann, el sentido como el medio y aún sin sentido es sentido -la forma de dos lados, mientras que una forma está vigente, la otra se encuentra marginada pero puede cambiar en cualquier momento por una reinterpretación (Luhmann, 1998) nos permite continuar la comunicación en el disenso que implica la posibilidad entre presente y futuro, entre presente y lo ausente, incluye la diferencia que en cuanto los horizontes espaciales cambien habrá un cambio de sentido, que puede explicar el sentido de una sentencia que revoca la anterior, por ejemplo, así, el presente se volverá pasado. Se trata de una modificación en los horizontes. (Luhmann, 1998: 93), en el ejemplo, en los horizontes interpretativos de los dos juzgadores. Y para el público en general, esta aparente contradicción no se basa en una explicación jurídica sino en una percepción moral, de sentido ético o incluso de justicia o injusticia, por tanto, el segundo juzgado revoca la anterior sentencia porque era injusta, por ser un juez bueno o malo, porque es corrupto, etcétera.

Con Luhmann, la diferenciación está reconocida, e incluso permite el cambio del código jurídico, lo que hoy es ilícito podrá ser declarado lícito en una reforma legal, una modificación constitucional, o incluso en una sentencia. La comunicación surgida del 
disenso crea espacios temporales donde existe la posibilidad de un cambio social. Algo que la sociedad en general, dinámica y atemporal exige del Derecho.

Los sentidos y sin sentidos de la interpretación de los contenidos del Derecho, como las resoluciones, sentencias, leyes, etcétera presentan, al mismo tiempo, un sentido y un sin sentido.

Un sentido jurídico dotado por los operadores del Derecho y un sentido dado por la sociedad, el punto central para divulgar adecuadamente a la Ciencia Jurídica entre el público no especializado es preguntarse si ese sentido ¿coincidirá? O tal vez, coincidamos más en los sin sentido, que en el sentido.

Por tanto, la complejidad de divulgar el Derecho va más allá de realizar intentos de información acerca del articulado de una ley, sino en transformarlo en códigos de lenguaje que doten de sentido. El reto se enfoca a un solo sujeto: el Divulgador.

El divulgador puede o no tener la categoría de operador jurídico, posee su propio horizonte que tal vez dificulte la dotación de sentido en el mismo sentido de la hermenéutica del Derecho.

\section{Hermenéutica y divulgación del Derecho}

Para Gadamer y Ricoeur, la hermenéutica es una pretensión del ser a través de la comprensión: "la comprensión lo es siempre en el modo de la representación y no de la mera presentación” (Estrada, 2019: 24).

Y ya que somos seres humanos con vivencias determinadas esto implica la creación de puntos ciegos debido al momento de interpretar:

También como intérprete de textos científicos tiene uno que contar normalmente con esta coexistencia de un uso terminológico y un uso corriente de una palabra. Los intérpretes modernos de los textos clásicos se inclinan muchas veces a desatender la importancia de este problema, porque el concepto resulta más artificial y por lo tanto más fijado en su moderno uso científico que en la antigüedad, en la que todavía se conocían pocos extranjerismos y términos inventados. (Gadamer,1975: 499)

El lenguaje jurídico como lenguaje técnico se vuelve riguroso y a través de sus propios métodos hermenéuticos crea realidades jurídicas que resuelve problemas comunes de las personas, el dilema con el Derecho, es que si bien los textos van dirigidos a una comunidad que ha aprendido el lenguaje técnico, las normas se dirigen y regulan comportamientos de los habitantes de una sociedad, el dilema es utilizar un lenguaje legal que parezca normal, eso da mucha incertidumbre a los juristas, porque el tecnicismo y la rigurosidad son propios de un Estado de Derecho, objetivo final de la cultura de la legalidad:

Si el léxico técnico del lenguaje legal es lo que lo hace riguroso y seguro, traducirlo-para hacerlo más accesible-al léxico común tendría un precio muy elevado, porque puede que el derecho lo comprendieran más los ciudadanos, pero a costa de dejar de comunicarse con rigor los juristas. (Pietro, 1996:118)

Entonces, además del horizonte del intérprete que divulga, en el caso del Derecho, debemos observar que el lenguaje utilizado no sea demasiado común porque el Derecho como disciplina de excesivo rigor expresivo y funcional (referente a los mecanismos propios 
de autorreferencia y construcción del contenido jurídico) No admite un uso corriente del lenguaje en la divulgación:

Laminar los recursos de la lengua y sus posibilidades expresivas en aras de un lenguaje jurídico más simple y funcional es cometer simpleza: si para conseguir lo simple (una mayor facilidad y pureza) se llega a incurrir en la simpleza (que pertenece a la categoría de lo estúpido) es preferible dejar las cosas como están. (Pietro, 1996:129)

La divulgación del Derecho me parece notoriamente trascendental y veo que, a pesar, de ser evidente la necesidad de una cultura de la legalidad en México, el tema no está explorado porque a los juristas les parece banal, sin embargo en situaciones imprevistas y notoriamente trascendentes que rompen el mundo conocido tal y como sucede con la pandemia del Covid-19, dar respuesta a los cuestionamientos de los sectores sociales acerca de los temas jurídicos que afectan su realidad, se vuelve una tarea esencial de las universidades. ${ }^{1}$

A partir del discurso de los Derechos Humanos como eje transversal de las distintas ramas del Derecho, comienza una era en la cual la divulgación del Derecho es más aceptada pero aún se utiliza un lenguaje demasiado riguroso. ${ }^{2}$

La divulgación del derecho resalta su función social: ser útil en la vida cotidiana y democrática de las sociedades, en este caso de la sociedad mexicana.

Así el Derecho como ciencia social implica una función cultural basada en el principio jurídico de <la ignorancia de la ley no exime de su cumplimiento $>$.

Por ello, uno de los problemas más relevantes de la Enseñanza del Derecho, además de la promoción de la investigación jurídica por parte del alumno, es el enfoque de una ciencia social con miras de divulgación útil al desarrollo de una nueva competencia: la participación ciudadana. Así se debe considerar una nueva labor de la enseñanza del Derecho en la creación de una nueva cultura de la legalidad en México.

Una manera de comenzar a divulgar sería fomentar las revistas jurídicas editadas por los estudiantes dentro las Universidades en México, en especial la Facultad de Derecho de la UNAM, como expresión de la creatividad del estudiante y el planteamiento de argumentos de forma escrita, estimulando la investigación y empujando la calidad de los resultados. (Boto, 2012).

\footnotetext{
${ }^{1}$ En México. ante la Pandemia del Covid-19, la Universidad Nacional Autónoma de México, mediante el Instituto de Investigaciones Jurídicas, ha elaborado la guía jurídica en colaboración con los Estándares Pro Bono México, la Fundación Appleseed México, A.C, el Centro Mexicano Pro Bono. A.C., la Fundación Barra Mexicana, A.C., el Consejo General de la Abogacía Mexicana, el ilustre y Nacional Colegio de Abogados de México, la Asociación Nacional de Abogados de Empresas, Colegio de Abogados A.C. disponible para su consulta en https://asesoría.juridicas.unam.mx . La Facultad Derecho de la misma Universidad puso a disposición del público en general el Observatorio de Asesoría Jurídica COVID-19 disponible en https://www.derecho.unam.mx/observatorio-juridico/

2 Un ejemplo lo encontramos en el siguiente artículo en una Revista de Derecho que se especializa en Divulgación y así se anuncia donde el mero título implica un lenguaje no dirigido a un público heterogéneo: "Dignidad Humana y Derecho Penal: Una difícil convergencia aproximación al contenido constitucional de la norma rectora..."
} 


\section{Información y Divulgación en los medios de comunicación: ¿la divulgación del Derecho es exclusiva de los expertos jurídicos o los periodistas pueden participar en ella?}

Para iniciar este apartado haré referencia al periodismo científico y la diferencia con el periodismo de divulgación.

Carlos Elías (2014), periodista español, en su libro Fundamentos de Periodismo Científico y divulgación mediática acota al mismo a la temática de las ciencias naturales y establece que los periodistas científicos no siempre hacen divulgación:

Deben matizarse las diferencias entre periodismo y divulgación científica, pues, aunque aspiren a un objetivo común: informar sobre ciencias naturales, y en muchos casos, compartan fundamentos y pautas de trabajo similares, sus enfoques de la información científica son muy distintos. La diferencia básica entre divulgación científica y el periodismo científico es que la divulgación la hacen siempre las fuentes (en este caso los científicos) y, por tanto, tiene como objeto beneficiar el entorno de la fuente. El periodismo científico a veces hace divulgación, pero no se conforma sólo con eso; también debe contextualizar sobre, por ejemplo, si socialmente merece la pena tal experiencia o si es necesario retirar financiación de un programa de ayuda a desfavorecidos para financiar un telescopio espacial. Por tanto, el periodista no busca beneficiar a la fuente sino a la sociedad. (Elías, 2014: 16)

Partiendo de lo anterior y de lo escuchado en las sesiones del periodista Humberto Musacchio (abril-septiembre, 2019) cuando menciona a las notas periodísticas como crítica hacia las políticas gubernamentales en el entendido de lo que beneficia o no a la sociedad, se puede afirmar que:

El periodismo desempeña diversas funciones en la sociedad contemporánea como fuente de información, análisis y comentario de los hechos de actualidad. No obstante, el objetivo básico de la mayoría de los periodistas es servir a la sociedad informando a la ciudadanía, interrogándose sobre el modo en que se ejerce el poder, favoreciendo al debate democrático y, con ello, contribuyendo al desarrollo político, económico, social y cultural. (Flores, 2014: 513)

Además de lo dicho en su ponencia el divulgador Javier Aranda Luna (abril-septiembre, 2019) quién resalta el valor de comunicar los resultados de la investigación desde la academia, aunque también como parte de una crítica social (concretamente él se refiere al Movimiento de 68). Me parece que, si existe diferencia entre el periodismo y la divulgación, tal como señala Elías, se tratan de objetivos distintos, aunque tal vez, en el punto de la crítica gubernamental se extrapole el sentido y pueda perderse el origen de la divulgación ya que, en lugar de comunicar los resultados de una investigación realizada por la fuente, se confunda con una crítica hacia el gobierno y se pierdan seguidores o auditorio.

Entonces, el periodismo científico rompe las reglas del periodismo que crítica al Gobierno, es un periodismo que da respiros o que mueve conciencias hacia temas como el ambiente, salud, entre otros, el lenguaje está orientado a la acción.

Un aspecto que me gustaría enmarcar en esta reflexión es el concepto de científico, en los inicios del periodismo se trata solo de temas de ciencias exactas, aún sin considerar al medio ambiente, adquirió sensacionalismo cuando se incluye la variable de daños ambientales, el cambio climático y la extinción de las especies, se transforma a un periodismo científico social:

...el periodismo científico tiene unas especiales particularidades que derivan en la circunstancia de que la labor del periodista científico no debe limitarse a ser un simple informador, sino que debe asumir 
una cierta función "formativa" (sic). De este planteamiento la información y la divulgación aparecen juntas, fusionadas y la actividad formativa que se desprende resulta connatural a la propia especialización. (Belenguer, 2003: 44)

Al periodismo científico extraído del mundo de las ciencias exactas como la biología o las matemáticas, se le incluye la cultura, las humanidades y las ciencias sociales, para convertirse en un periodismo divulgador:

Lo que entendemos por Divulgación Científica es precisamente esto: toda actividad de explicación y difusión de los conocimientos, la cultura y el pensamiento científico y técnico, bajo dos condiciones, con dos reservas: la primera es que estas explicaciones y esa difusión del pensamiento científico y técnico sean hechas fuera de la enseñanza oficial o de enseñanza equivalentes....La segunda reserva es que estas explicaciones extraescolares no tengan por fin tomar especialistas, ni tampoco perfeccionarlos en su propia especialidad, ya que, por el contrario, reivindicamos completar la cultura de los especialistas fuera de su especialidad. (Belenguer, 2003:45)

Así, el divulgador no solo es el investigador que realiza el estudio o la fuente, sino también puede ser una persona, un periodista con habilidades o competencias de explicación y difusión de conocimientos en un lenguaje dirigido a un público no especializado. Un ejemplo es el Pint of Science que se realiza en 73 ciudades españolas, donde científicos presentan sus investigaciones en forma sencilla a través de experimentos, demostraciones científicas, espectáculos o proyecciones en bares y pubs y cuyo formato se está replicando en Mazatlán, Sinaloa, en una iniciativa llamada "Pisteando con Ciencia" (Michel, 2019).

El lenguaje, formato y la extensión temporal del discurso, lo que tal vez, haga de una experiencia de divulgación periodística un caso exitoso respecto a la divulgación realizada desde las universidades, ${ }^{3}$ ya que el investigador tenderá a utilizar el mismo lenguaje de su investigación y se enfocará a un público con niveles universitarios aunque no sean en el campo de las disciplina en la cual desarrolla su investigación, tal y como pasa, con los literatos que divulgan a otros autores, escriben sobre ellos, pero realizando un análisis que bien puede ser objeto de una tesis de posgrado.

Los formatos utilizados por las universidades suelen ser medios escritos, conferencias (en muchas ocasiones leídas por el ponente, efecto muy adverso cuando el auditorio es un público general), presentaciones digitales planas, sin animaciones, fotografías e infografías estáticas. Videos y audios de duración mayor a una hora, lo cual hace que solo público especializado o por especializarse sea el único auditorio interesado en estos materiales; como un ejemplo de recurso abierto interactivo con lenguaje de divulgación es el video: "Reflexión sobre competencia económica, consumidores y comercio exterior", que se encuentra disponible en el sitio de Facebook de la Facultad de Derecho de la UNAM (Witker, J. A., Cázares, C., Arley, D., 2020).

Tal vez el temor a ser considerados por los pares como vulgares ${ }^{4}$ haga de la actividad de divulgación de la ciencias sociales y humanidades dentro de las universidades una actividad dirigida a un público con necesidades educativas muy puntuales, como estudiantes de las disciplinas, tesistas, investigadores, estudiantes de posgrado, profesores. En cambio, en la

\footnotetext{
${ }^{3}$ Uno de los ejemplos es Coordenadas 2050 Proyecto de la Coordinación de Humanidades UNAM.

${ }^{4}$ Del lat vulgaris: 3. Adj. Que es impropio de personas cultas o educadas. RAE, 2019.
} 
divulgación derivada del periodismo científico cultural, el público sea un auditorio más diverso.

A nivel de escuelas de Derecho, existen dos opciones: Realizar proyectos de divulgación del derecho administrados, diseñados y alimentados por materiales audiovisuales hechos por los estudiantes de Derecho, supervisados por los docentes. ${ }^{5}$

La segunda opción es fomentar proyectos de divulgación dirigidos a público en general con la finalidad de vincular a las Facultades de Derecho y la sociedad en general. ${ }^{6}$

\section{La humildad académica}

La importancia de estructurar una metodología flexible de investigación que incluya el uso de tecnologías de información y comunicación con la finalidad de divulgar desde la academia se basa no solo en una necesidad social de apoyo a la comunidad, sino también, incluyen habilidades académicas, es decir, al incluir a la divulgación como un objetivo de las investigaciones de las Facultades de Derecho, se fortalece el pensamiento crítico para la resolución de problemas cotidianos (Difabio, 2005:178).

Además, de impulsar el pensamiento crítico, al enseñar a los estudiantes de Derecho, que uno de los objetivos de la investigación jurídica debe ser la divulgación del derecho y motivarlos a escribir para auditorios no expertos en Derecho, fomenta su autoestima y confianza según Siegel (1990) (como se citó en, 2005:173), el divulgador se atreve a exponerse ante un público en general, se presenta ante sus no-pares, los sujetos a quienes se dirigen las normas, a sus posibles clientes. Se trata de un ensayo-error de vinculación con el sujeto real de la práctica del Derecho, sin que exista una responsabilidad mayor como lo hay en las clínicas jurídicas donde se asesora o realizan diversos procesos jurídicos bajo supervisión académica.

No solo los estudiantes de Derecho deberán ensayar a convertirse en divulgadores, también, los docentes y los investigadores, para ello, me parece necesario aprehender la información científica social o humanística y desprenderse de lo que he denominado: 'vanitas universitaria', 7 partir del principio de humildad y sencillez en uno mismo para entender que los títulos, los libros, los artículos que distinguen los méritos de un investigador a otro, fuera de la academia no poseen el mismo peso y los auditorios no

\footnotetext{
${ }^{5}$ Al respecto el Proyecto para el Mejoramiento de la Enseñanza del Derecho PE304116 autorizado por la Dirección General del Personal Académico y la Facultad de Derecho, UNAM fue pionero en el marco del fomento de la divulgación del Derecho. Se puede consultar en buhotica.com y en Cázares Sánchez Cristina (abril, 2017) El proyecto Derecho de Todos: comunidad de aprendizaje con el uso de TIC. Ponencia escrita presentada en el Congreso CEEAD Educación Jurídica 2018 organizado por el CEEAD, CIDE, ITAM, U de G, UDEM, ITESO, IBERO, ontre otras. Recuperado de https://congresoceead.mx/pdf.js/web/viewer.html?file=/assets/pdfs/171207_Memorias_CC17.pdf

${ }^{6}$ La Suprema Corte de Justicia de la Nación en México cuenta con material didáctico consistente en publicaciones de divulgación para ciudadanos de diversas edades, disponibles en www.scjn.gob.mx

${ }^{7}$ Dentro del mundo de los abogados litigantes, existe una costumbre, si un abogado-académico se ostenta como Doctor o Maestro dentro de un Juicio, normalmente el Juez, la parte contraria, hasta los archivistas suelen ponerle trabas dentro del proceso toda vez que ven como un acto de vanidad el ostentar los grados académicos pues la gran mayoría de los litigantes solo tiene el de licenciado en Derecho. Así la mayoría de los que estamos dentro de la Academia y llegamos a litigar, dejamos los doctorados para el aula y nos volvemos licenciados en los Tribunales.
} 
especializados no acuden a las actividades de divulgación buscando conocer al académico de renombre, como si sucede en la Universidad, donde un estudiante o profesor va a la conferencia magistral buscando conocer al autor del libro base de sus clases; por el contrario, tal vez con el público en general tengamos los académicos una sola oportunidad de ser oídos y vencidos por la crítica.

Denostar que en el ejercicio de divulgar los contenidos de una investigación ya sea por los medios de comunicación y convertirnos los docentes, investigadores y estudiantes en periodistas 'jurídicos' implica el acto creativo y más libre en el que podamos académicamente expresarnos, se trata de una muestra del conocimiento libre y humilde: "Por eso todo otro acto de conocer, desde el mínimo racional, el que se agota presentando un objeto conocido, es una manifestación del acto de ser personal y, por ende, muestra la humildad personal"(Sellés, 2019: 94).

El reto es redactar para un público general, captar su atención, comunicarse y exponer la importancia de los temas jurídicos para la sociedad.

\section{Conclusión}

La divulgación del Derecho debe incluirse dentro de los programas de estudio de las Facultades de Derecho, en todos los niveles, desde la licenciatura hasta el Doctorado, y fomentarse como competencias en los estudiantes, en dos vertientes: formativas, mientras se encuentran en las aulas (presenciales o virtuales): la investigación y las digitales donde muestren su creatividad en la realización de materiales idóneos para ser difundidos en los medios de comunicación en formatos digitales susceptibles de ser consultados por el público en general.

Y competencias para el trabajo, con el ejercicio continuo de divulgar lo investigado como tareas académicas, desarrollan la empatía con su futuro cliente que no necesariamente poseerá un grado académico pero que indudablemente acudirá al futuro abogado porque el Derecho ha transformado su realidad. 


\section{Fuentes de Consulta}

Arráez, M., Calles, J. y Moreno de Tovar, L. (diciembre, 2006). La Hermenéutica: una actividad interpretativa. Sapiens. Revista Universitaria de Investigación, 7(2), Universidad Pedagógica Experimental Libertador, Caracas, Venezuela. pp. 171-181. Disponible en https://www.redalyc.org/pdf/410/41070212.pdf

Belenguer Jané, M. (2003). Información y divulgación científica: dos conceptos paralelos y complementarios en el periodismo científico. Estudios sobre el Mensaje Periodístico, pp. 43-53.

Boto Álvarez, A. (2012). El potencial de las revistas jurídicas, Revista electrónica "Docencia y Derecho", No. 4. Facultad de Derecho y C.C. E.E. y Empresariales, Córdoba. Pp. 15 . Recuperado de https://www.uco.es/docencia_derecho/index.php/reduca/article/view/59

Coordinación de las Humanidades. (abril-septiembre 2019). Diplomado en Divulgación de las Humanidades 2019. UNAM, México.

Difabio De Anglat, H. (2005) The Critical Thinking Movement and the intellectual education, Estudios sobre Educación, 2005, 9, Pp. 167-187.

Elías, C. (2014). Fundamentos de periodismo científico y divulgación mediática, edición digital, Alianza Editorial, Madrid.

Estrada Castro, L. J. (2019). Conceptos de Hermenéutica. Diplomado en Divulgación de las Humanidades. Coordinación de Humanidades, UNAM. Presentación de diapositivas.

Flores Vivar, J. M. (julio-noviembre, 2014). El periodismo como disciplina del conocimiento y el valor academicista para una profesión de calidad. Prisma Social, núm. 12, pp. 506-543. Recuperado

de http://www.isdfundacion.org/publicaciones/revista/numeros/12/periodismodivulgacion-cientifica.html

Gadamer, H.-G. (1975). Verdad y Método I. Décima edición. Trad. Ana Agud Aparicio y Rafael de Agapito. España 2003.

Habermas, J. (1987) Teoría de la Acción comunicativa. Volumen 2: “Crítica de la razón funcionalista". Taurus. Madrid. 
Luhmann, N. (1998). Sistemas Sociales. Lineamientos para una teoría general. Anthropos Editorial/Universidad Iberoamericana/CEJA, Pontificia Universidad Javeriana. España.

Michel. (7 de junio de 2019). Pistear y aprender es posible: proyecto de divulgación enseña desde bares. plumasatomicas.com. Recuperado de https://plumasatomicas.com/noticias/ciencia/pistear-y-aprender-es-posibleproyecto-de-divulgacion-ensena-desde-bares/

Pietro De Pedro, J. (mayo-agosto de 1996). Lenguaje Jurídico y Estado de Derecho. Revista de Administración Pública, Núm. 140, 116-129.

Portales, M. (20 de mayo de 2019). La ciencia se cuela en los bares. El País.com. Recuperado de https://elpais.com/elpais/2019/05/16/ciencia/1557996809_313933.html

Sellés Dauder, J. (2019). La humildad según Leonardo Polo. Contrastes. Revista Internacional de Filosofía, vol. XXIV-No3 (2019), pp. 91-104. ISSN: 1136-4076 Departamento de Filosofía, Universidad de Málaga, Facultad de Filosofía y Letras Campus de Teatinos, E-29071 Málaga, España.

Witker, J. A., Cázares, C., y Arley, D. (abril de 2020). Reflexión sobre competencia económica, consumidores y comercio exterior. [Archivo de video]. Recuperado en https://www.facebook.com/DerechoUNAM/videos/154825019286540/ 